Article

\title{
Peculiarities of the Acoustic Wave Propagation in Diamond-Based Multilayer Piezoelectric Structures as "Me1/(Al,Sc)N/Me2/(100) Diamond/Me3" and “Me1/AlN/Me2/(100) Diamond/Me3" under Metal Thin-Film Deposition
}

\author{
Gennady Kvashnin ${ }^{1}$ (D), Boris Sorokin ${ }^{1}\left(\mathbb{D}\right.$, Nikita Asafiev $^{1,2}(\mathbb{D})$, Viacheslav Prokhorov ${ }^{1,2, *(\mathbb{D})}$ \\ and Andrei Sotnikov ${ }^{3,4}$
}

check for updates

Citation: Kvashnin, G.; Sorokin, B.; Asafiev, N.; Prokhorov, V.; Sotnikov, A. Peculiarities of the Acoustic Wave Propagation in Diamond-Based Multilayer Piezoelectric Structures as “Me1/(Al,Sc)N/Me2/(100) Diamond/Me3" and "Me1/AlN/ Me2/(100) Diamond/Me3" under Metal Thin-Film Deposition.

Electronics 2022, 11, 176. https:// doi.org/10.3390/electronics11020176

Academic Editor: Antonio Di Bartolomeo

Received: 30 November 2021

Accepted: 5 January 2022

Published: 7 January 2022

Publisher's Note: MDPI stays neutral with regard to jurisdictional claims in published maps and institutional affiliations.

Copyright: (C) 2022 by the authors. Licensee MDPI, Basel, Switzerland. This article is an open access article distributed under the terms and conditions of the Creative Commons Attribution (CC BY) license (https:// creativecommons.org/licenses/by/ $4.0 /)$.
1 Federal State Budgetary Institution "Technological Institute for Superhard and Novel Carbon Materials" (FSBI TISNCM), 108840 Troitsk, Russia; genmih@yandex.ru (G.K.); bpsorokin1953@yandex.ru (B.S.); asafev.no@phystech.edu (N.A.)

2 Moscow Institute of Physics and Technology, National Research University, 141701 Dolgoprudny, Russia 3 Leibniz Institute for Solid State and Materials Research, 01069 Dresden, Germany; a.sotnikov@ifw-dresden.de 4 Ioffe Institute, 194021 St. Petersburg, Russia

* Correspondence: pvm@tisnum.ru

\begin{abstract}
New theoretical and experimental results of microwave acoustic wave propagation in diamondbased multilayer piezoelectric structures (MPS) as "Me1/(Al,Sc)N/Me2/(100) diamond/Me3" and "Me1/AlN/Me2/(100) diamond/Me3" under three metal film depositions, including the change in the quality factor $Q$ as a result of Me3 impact, were obtained. Further development of our earlier studies was motivated by the necessity of creating a sensor model based on the above fifth layered MPS and its in-depth study using the finite element method (FEM). Experimental results on the change in operational checkpoint frequencies and quality factors under the effect of film deposition are in satisfactory accordance with FEM data. The relatively small decrease in the quality factor of diamond-based high overtone bulk acoustic resonator (HBAR) under the metal layer effect observed in a wide microwave band could be qualified as an important result. Changes in operational resonant frequencies vs. film thickness were found to have sufficient distinctions. This fact can be quite explained in terms of the difference between acoustic impedances of diamond and deposited metal films.
\end{abstract}

Keywords: high overtone bulk acoustic resonator (HBAR); quality factor; microwave band; ultrasonic sensor device; thin- and ultrathin-film deposition; single-crystalline diamond; aluminum nitride; aluminum-scandium nitride

\section{Introduction}

Currently, the development of acousto-electronic sensors intended for various applications such as biology, fine physicochemical studies, analysis of the gas composition of the atmosphere, and detection of explosives is in active progress. There are many approaches to solving this problem. Using modern technologies, one can detect the adsorption of a single gas molecule. For example, in [1], the concentration of various gases was recorded at the level of one ppb. This high resolution was achieved due to the unique sensory properties of graphene. Most of the known acoustic sensory methods register changes in the resonant frequency of the device as an oscillating plate, a cantilever made of silicon, and resonators based on the surface (SAW) or bulk acoustic waves (hereinafter referred to as the BAW resonator). The record sensitivity of mass measurement of several attograms was demonstrated in $[2,3]$. Using a cantilever's frequency shift vs. mass load, the mass value of 
the smallpox virus of about $12 \mathrm{fg}$ was found [2]. The authors in [4] described an application of the nanoelectromechanical system (NEMS) device to ultrasensitive mass detection and measured the Au film mass deposition with the resolution of some attograms. Such sensors had to satisfy the high demands of the experiment arrangement such as a high vacuum and precise temperature control at low temperatures. A special problem is the one-time use of the NEMS sensory element. In addition, there has always been a question about the complicated calibration procedure of that device. Thus, the search for analogs, which are simpler and more convenient in a practical sense, remains open. Acousto-electronic sensor devices have some advantages in this field.

The most known and widely employed sensor is the so-called quartz crystal microbalance (QCM) which was first developed by Sauerbrey [5]. This device consists of a quartz plate with metal electrodes deposited on the main surface, i.e., there is a piezoelectric resonator with the longitudinal or shear bulk acoustic waves as operational modes. The reduced mass sensitivity of such devices changes from $10^{8}-10^{10} \mathrm{~Hz} \cdot \mathrm{cm}^{2} / \mathrm{g}[6,7]$. The authors of [6] showed that the relative frequency shift under the large mass load deviated from the linearly proportional dependence and depended on the acoustical impedance ratio of quartz and deposited film. The QCM device has been employed for studying metal film deposition, electroplated alloy etching rate, oxidation processes of metal film, and the physical properties of the solid-liquid interfacial layer in situ [8]. Other well-known sensor schemes are based on the properties of SAW resonators. Their sensitivity has the typical value of about $2.5 \times 10^{-9} \mathrm{~Hz} \cdot \mathrm{cm}^{2} / \mathrm{g}$ [9]. The film bulk acoustic resonator (FBAR) as a sensor has the typical value of mass sensitivity about $3.6 \times 10^{-6} \mathrm{~Hz} \cdot \mathrm{cm}^{2} / \mathrm{g}[10]$. A given device showed a thickness sensitivity to the deposition of the Ti film of about $1.6 \mathrm{~nm}$, achieved at an operating frequency of about $2442 \mathrm{MHz}$. Furthermore, small sensory devices based on microelectromechanical systems (MEMS) have been developed. For example, a MEMS FBAR-based mass sensor built on a micromachined silicon nitride diaphragm and operating in vapor and liquid had a mass sensitivity of $726 \mathrm{~cm}^{2} / \mathrm{g}$, which is about 50 times than that of a typical quartz crystal microbalance [11]. However, the quality factor $Q$ of such a device in air was about 200-300 at $1 \mathrm{GHz}$ and dropped to about 40 at $2 \mathrm{GHz}$. This would lead to a drop in the sensor's sensitivity. In [12], the possibility of using a thin-film acoustic resonator with a Bragg reflective grating as a sensor of organic films was considered. Operating frequencies of $6-8 \mathrm{GHz}$ were used. The authors [13] investigated the use of an FBAR sensor with a shear operating mode for studying the properties of liquids. At a resonant frequency of $1.25 \mathrm{GHz}$ in water, the $Q$-factor of the resonator decreased to 150 from the value of 300 in air. It should be noted that both membrane FBAR sensors and those developed using MEMS or NEMS technology have one common disadvantage in that they cannot be reused.

Mansfel'd et al. [14] proposed the high overtone bulk acoustic resonator (HBAR) for the spectroscopy of metal film properties. The authors of [15] proposed an experimental and model investigation of the sensory properties of $\mathrm{HBAR}$ structures as " $\mathrm{AlN} / \mathrm{SiO}_{2}$ " and

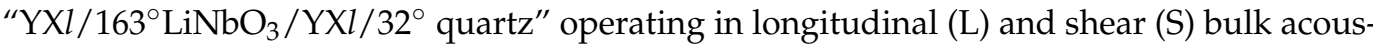
tic modes, respectively. The main objective consisted of improving the HBAR's gravimetric (mass) sensitivity. The peculiarities of BAW reflection and penetration into deposited films were established. However, in our opinion, the HBAR's sensory properties were insufficiently studied. Hence, owing to HBAR's high quality factor in the microwave band, one can get increasing sensitivity taking into account the high operational frequency which will sufficiently exceed that in SAW- or FBAR-based sensors. However, such an opportunity can only be realized if the substrate's material possesses low acoustic attenuation in the microwave frequency band, e.g., diamond [16]. Here, diamond's excellent acoustic properties were investigated in the microwave band up to $20 \mathrm{GHz}$. It is shown that the diamond-based HBAR's quality factor of about 12,500 at $~ 20 \mathrm{GHz}$ remained at such a high value only owing to Landau-Rumer's mechanism of microwave acoustic attenuation in diamond [17]. The L-BAW excitation of the diamond-based HBAR in the extremely high frequency band up to $40 \mathrm{GHz}$ was published in [18]. A quality factor of $\sim 10,000$ at $40 \mathrm{GHz}$ was obtained. 
Recently, we obtained a Patent RF dedicated to the diamond-based microwave acoustic mass sensor [19]. Earlier, by means of diamond-based HBAR as a sensor, the deposition of thin and ultrathin metal films such as Mo, Al,Sc, and Pt was experimentally studied [20-22]. A new useful approach for analyzing the wave propagation in piezoelectric nano-size plates under special boundary conditions was proposed in [23]. According to [24], in the future, super-thin films, smart materials, and smart nanodevices will replace macro-sized elements in conventional acoustic devices. Thus, to design and build such novel elements, it is necessary to have models of acoustic wave propagation in small-scale environments while taking into account several factors.

This work makes the following contributions: (i) the experimental $Q$-factor's dependences on the film thickness are introduced and discussed; (ii) the HBAR-based sensor model is proposed and studied in detail using FEM; (iii) a complete explanation of the peculiarities of the BAW propagation in diamond-based MPSs under the influence of a certain metal film deposition is provided.

Therefore, the main objective of this work was concerned with a detailed study of the properties and opportunities of acousto-electronic sensors based on microwave HBAR with a diamond substrate, including a model visualization of acoustic wave propagation and the conditions of optimal sensitivity.

\section{Materials and Methods}

The HBAR-based sensors were realized using the "Me1/AlN/Me2/(001) diamond/Me3" or "Me1/(Al,Sc)N/Me2/(001) diamond/Me3" multilayered piezoelectric structures (MPS). Here, Me1 and Me2 are metallic electrodes such as Al, Mo, or Pt, and Me3 is the deposited metal film to be studied. The technology of the "Al/AlN/Mo/diamond" HBAR processing was described in detail in [21]. As a substrate, plates made of type Ila synthetic singlecrystalline diamond produced by FSBI TISNCM were applied. Piezoelectric films of aluminum nitride $\mathrm{AlN}$ and aluminum-scandium nitride $(\mathrm{Al}, \mathrm{Sc}) \mathrm{N}$ were used in the thinfilm piezoelectric transducers (TFPT) as Me1/ $\mathrm{AlN} / \mathrm{Me} 2$ or Me1/(Al,Sc)N/Me2 to excite the longitudinal bulk acoustic wave (L-BAW). However, the application of aluminumscandium nitride leads to more effective electromechanical excitation of L-BAW as an HBAR operational mode even in the extremely high-frequency band [18]. Figure 1 represents an example of such a sensory element based on the MPS "Pt/(Al,Sc)N/Pt/(001) diamond" (Sc content $15 \%$ ). There, one can see the four independent HBARs distinguished by the area of an active zone, hereafter titled an aperture. One of them, with the aperture of 10,000 sq. microns, was used as sensor \#C. The main parameters of the sensory elements studied are given in Table 1.

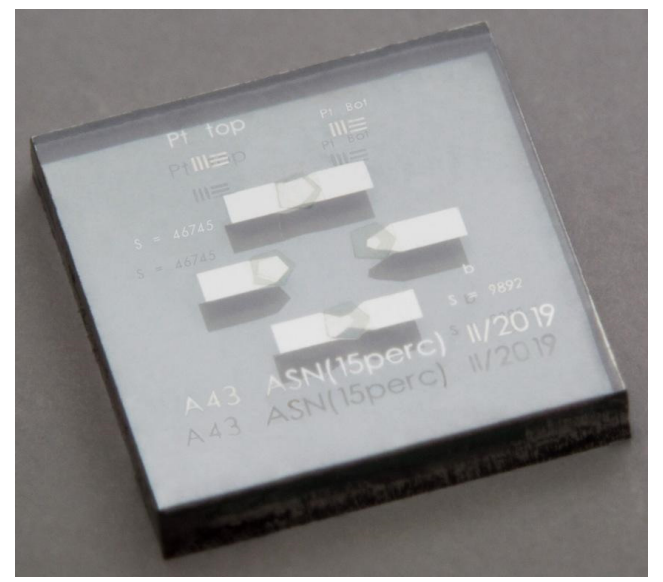

Figure 1. Sensory element based on the " $\mathrm{Al} /(\mathrm{Al}, \mathrm{Sc}) \mathrm{N} / \mathrm{Mo} /(001)$ diamond" multilayer piezoelectric structure. 
Table 1. Properties of HBARs in utilized sensors.

\begin{tabular}{cccc}
\hline Sensor Properties & Sensor \#A & Sensor \#B & Sensor \#C \\
\hline Top electrode material/thickness (microns) & $\mathrm{Al} / 130$ & $\mathrm{Al} / 131$ & $\mathrm{Pt} / 122$ \\
Bottom electrode material/thickness (microns) & $\mathrm{Mo} / 136$ & $\mathrm{Mo} / 159$ & $\mathrm{Pt} / 105$ \\
Piezoelectric layer thickness (nm) & 1120 & 930 & 1013 \\
Sc percentage in piezoelectric layer $\mathrm{Al}_{1-x} \mathrm{Sc}_{x} \mathrm{~N}$ & 0 & 0 & 13 \\
Diamond thickness (microns) & 482 & 501 & 629 \\
Area of the aperture (sq. microns) & 20,000 & 47,000 & 10,000 \\
\hline
\end{tabular}

To evaluate how different acoustic impedance affects the performance of a diamondbased sensor, three metals with widely varying acoustic properties were selected. A comparison of the acoustic characteristics of studied metals, including diamond, is presented in Table 2. Here, $Z=\rho V$, where $\rho$ is the material density, and $V$ is the L-BAW phase velocity.

Table 2. Properties of diamond and metals chosen for experiment.

\begin{tabular}{ccccc}
\hline Material and Acoustic Properties & Sc & Mo & Pt & Diamond \\
\hline Density $\rho, \mathrm{g} / \mathrm{cm}^{3}$ & 3.0 & 9.8 & 21.4 & 3.5 \\
L-BAW phase velocity $V, \mathrm{~m} / \mathrm{s}$ & 5590 & 6430 & 3830 & $17,542 /[100]$ \\
Acoustic impedance $Z, 10^{6} \mathrm{~kg} / \mathrm{m}^{2} \cdot \mathrm{s}$ & 16.74 & 63 & 84.7 & 61.7 \\
\hline
\end{tabular}

The metal films were deposited on the free side of the diamond substrate by the AJA Orion 8 magnetron sputtering equipment, which was used to fabricate the HBARs themselves. The number of layers varied from six to 40 in different approaches. The substrate holder was rotated with an angular speed of $1 \mathrm{rad} / \mathrm{min}$. In the case of thin layers, $h<20 \mathrm{~nm}$, the angular speed was increased to $2 \mathrm{rad} / \mathrm{min}$. The unevenness of the thickness of the deposited films was less than $5 \%$ over the entire surface of the substrate holder. The mean film growth rate was equal to $\sim 100 \mathrm{~nm} / \mathrm{h}$.

The thickness of deposited films in the sputtering process was controlled by a QCM sensor installed in the magnetron chamber. For more precise thickness measurement, the atomic force microscope (AFM) method for accompanying Si samples was used. The metal was sputtered using the same process as for the HBAR sensor. The measurement uncertainty of the AFM method was from $10 \mathrm{~nm}$ to $3 \mathrm{~nm}$. When the thickness of films was thinner than $5 \mathrm{~nm}$, it was estimated from the deposition time with linear interpolation using a known growth rate.

When a metal film was sputtered, the sensor was extracted from the magnetron chamber and brought to the probe station. After that, the shift of the overtone's resonant frequency and quality factors were measured. The process of the frequency response measurement of HBAR by the E5071C Agilent network analyzer was described in detail in [18]. The operational checkpoint frequencies were selected for every sensor separately, and the overtones with the highest quality factor or highest impedance response were prioritized. When the measurement for the $n$-th layer was over, the sensor was brought back to the magnetron chamber, and layer $n+1$ was sputtered.

To explain the data obtained, a computer simulation of acoustic wave propagation in MPSs using the COMSOL Multiphysics software was executed. For 2D MPS modeling, the diamond substrate and deposited films with a width of $10 \mu \mathrm{m}$ were used (Figure 2). This choice made it possible to reduce the number of calculations without a noticeable deviation from the qualitative and quantitative results of the experiment. Periodic type boundary conditions were applied. The maximum size of the grid cell did not exceed 1/10 of the elastic wavelength within a given particular layer. 


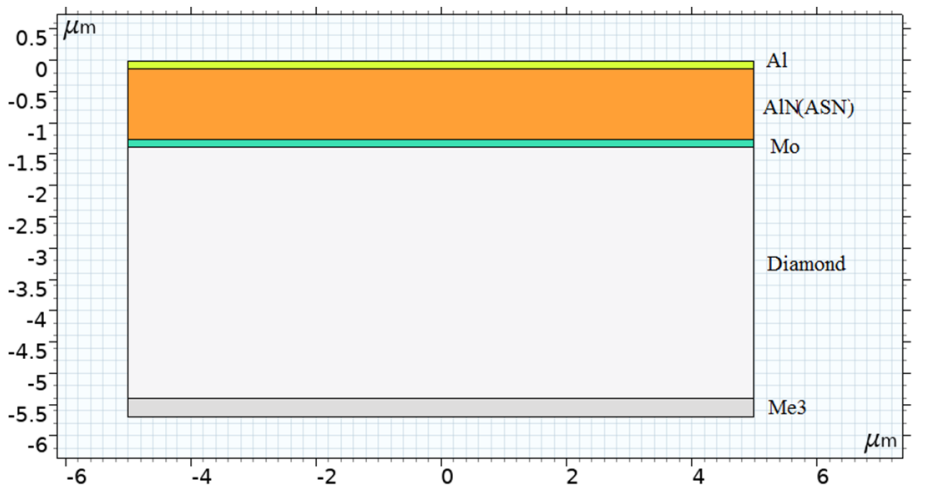

Figure 2. Model of diamond-based MPS. The layer thicknesses are shown on an arbitrary scale.

\section{Results}

The enhanced experimental data, including the relative frequency shifts and quality factors vs. the thickness of a deposited film, are presented in Figures 3-5. Here, the results of finite element modeling by COMSOL Multiphysics software are also shown. In FEM calculations, the actual thicknesses of all the piezoelectric and metal films and diamond substrates as in the experiment were applied. The thickness ranges of film deposition (in $\mathrm{nm}$ ) were changed within 0-950 for scandium, 0-390 for molybdenum, and 0-160 for platinum. The choice of operational acoustic overtones was fulfilled under the criterion of a maximal $Q$-factor of an HBAR at the specified resonant frequencies. The basis is that the frequency response of an HBAR is closely concerned with the TFPT frequency dependence, where the minimal and maximal $Q$ values should be observed in the vicinity of the points where the conditions $d \approx n \frac{\lambda}{4}$ or $d \approx m \frac{\lambda}{2}$ are fulfilled, respectively. Here, $d$ is the TFPT thickness, $\lambda$ is the wavelength in piezoelectric material, and $n=1,3, \ldots ; m=1,2, \ldots$ For more detail, see [20]. An example of a mentioned dependence is presented in Figure 5e. Owing to the high $Q$ values, this HBAR can be used as a sensory element in the frequency range from $1 \mathrm{GHz}$ up to $20 \mathrm{GHz}$.

The dependencies of frequency shift vs. the thickness of the different metal films change as a function of the relation between the acoustic impedances of diamond and a given film material. Therefore, one should consider three situations: (1) the metal Sc obeys the inequality $Z_{\text {diam }}>Z_{\text {Sc }}$; $(2)$ the metal Mo obeys the approximate equality $Z_{\text {diam }} \approx Z_{\text {Mo }}$; (3) the metal Pt obeys the inequality $Z_{\text {diam }}<Z_{\mathrm{Pt}}$.

Figure $3 a, b$ represent the relative frequency shift and quality factor vs. the Sc film thickness measured for the microwave acoustic overtones taken at four operational frequencies of $0.82 \mathrm{GHz}, 2.15 \mathrm{GHz}, 2.73 \mathrm{GHz}$, and $5.79 \mathrm{GHz}$ of sensor \#A. There, one can see the complicated dependencies of $\Delta f / f=f(h)$ and $Q=f(h)$ at the $2.15 \mathrm{GHz}$ overtone and higher (Figure $3 a, b)$. Figure $3 c-f$ demonstrate the model distribution of the L-BAW vertical Y-components of elastic displacements taken at unusual points as the inflections of curves $\Delta f / f=f(h)$ and extremums of the $Q=f(h)$ dependencies. The sensor's locations are shown only on the bottom side of an HBAR, including the Me3 deposited film. The red and blue patterns denote that the displacements were directed up or down, respectively. Thus, the distance between the corresponding red and blue points coincides with the $\lambda / 2$ value of the L-BAW in a given material at a selected frequency. Analyzing Figure $3 \mathrm{~d}-\mathrm{f}$, one can say that, at the resonant frequency of $5.79 \mathrm{GHz}$, the $\lambda / 4, \lambda / 2$, and $3 \lambda / 4$ values fit within the Sc thicknesses as $250 \mathrm{~nm}, 500 \mathrm{~nm}$, and $750 \mathrm{~nm}$, respectively. When $h=\lambda / 4$, the part of the L-BAW energy that penetrates into the deposited film reflects from a free film surface. As a result, the acoustic wave passes exactly a half wavelength within a film. This wave then interferes in the antiphase with a wave reflected out of the film-diamond interface. Hence, the interference minimum and a decrease in reflectance should be observed. This phenomenon is quite similar to the single-layer antireflection coating in optic devices. In the condition $h=\lambda / 2$, a bulk acoustic wave penetrates from diamond into the Sc film and then reflects from its free surface. Rereflection within the Sc free surface and Sc-diamond 
interface is also observed. In this case, the resonance phenomenon is directly observed in the deposited film. It should be emphasized that the $Q$-factor is approximately the same as in the initial state of the sensor $(h=0)$. In contrast, Figure $3 c$ shows the absence of such peculiarities at $0.82 \mathrm{GHz}$ because $\lambda>>h$.

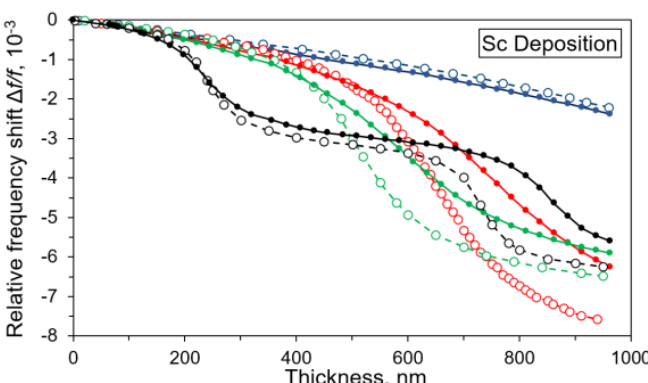

$-00.82 \mathrm{GHz}$ Mod. $\rightarrow-0.82 \mathrm{GHz}$ Exp. $-0.2 .15 \mathrm{GHz}$ Mod. $\rightarrow-2.15 \mathrm{GHz}$ Exp. $2.73 \mathrm{GHz}$ Mod. $\rightarrow-2.73 \mathrm{GHz}$ Exp. $-\mathrm{o} 5.79 \mathrm{GHz}$ Mod. $\rightarrow 5.79 \mathrm{GHz}$ Exp.

(a)

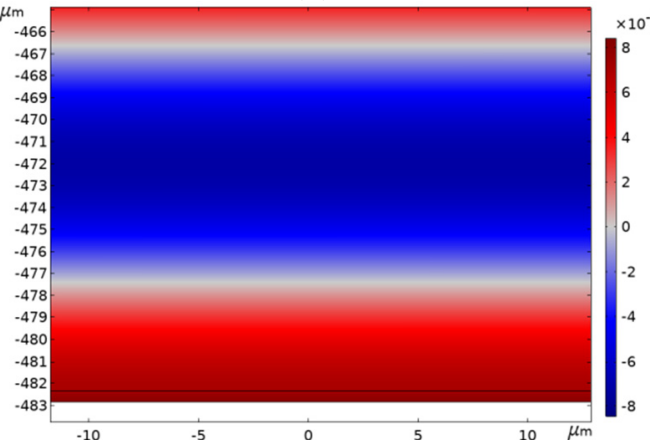

(c)

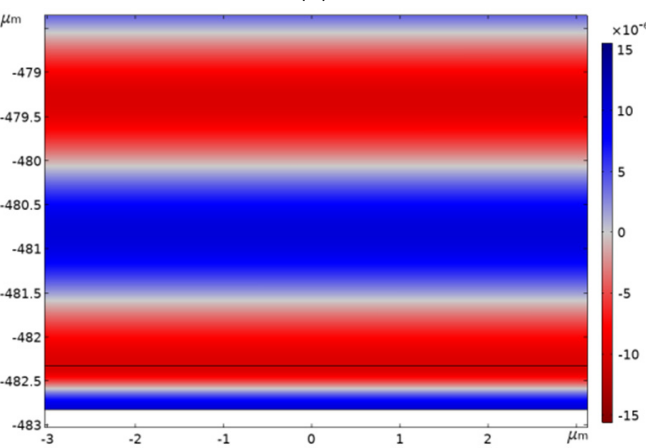

(e)

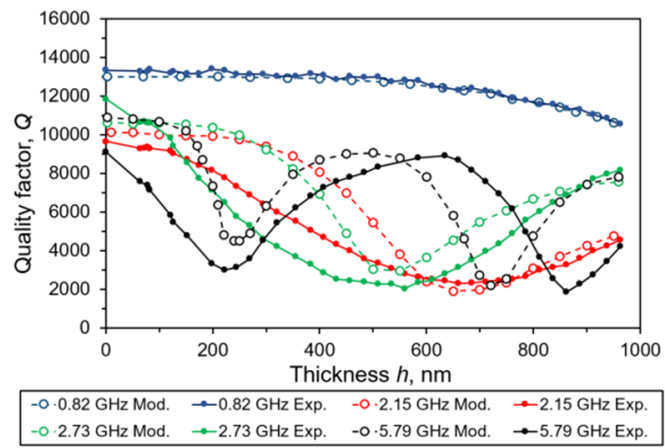

(b)

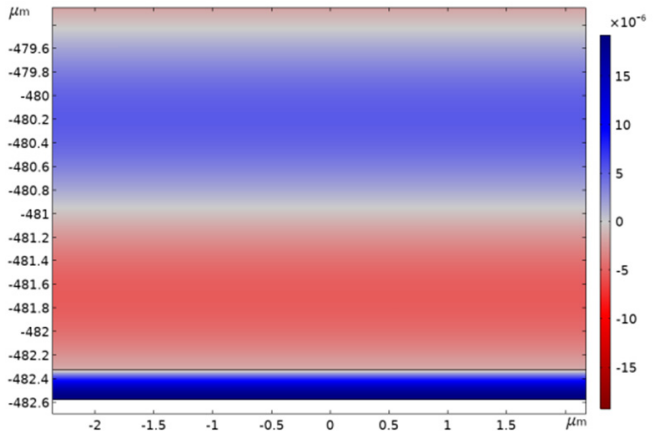

(d)

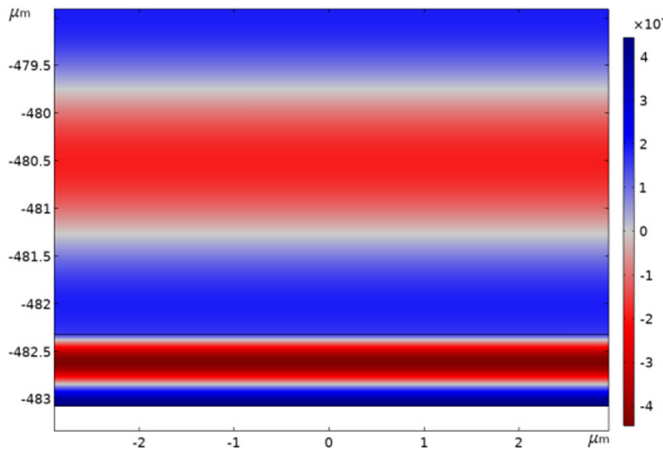

$(\mathbf{f})$

Figure 3. Relative frequency shift (a) and quality factor (b) vs. the Sc film thickness measured for the microwave acoustic overtones taken at four operational frequencies of sensor \#A. Model distribution of the L-BAW vertical Y-component of elastic displacements when $\mathrm{h}=500 \mathrm{~nm}$ at $0.82 \mathrm{GHz}$ (c), h $=250 \mathrm{~nm}(\mathbf{d}), 500 \mathrm{~nm}(\mathbf{e})$, and $750 \mathrm{~nm}(\mathbf{f})$ at $5.79 \mathrm{GHz}$. 


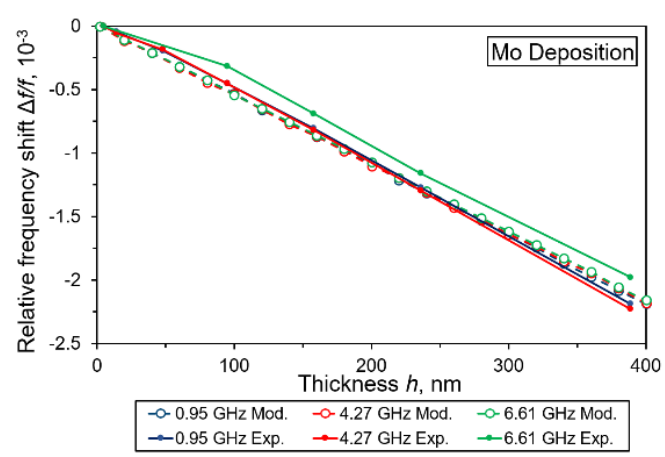

(a)

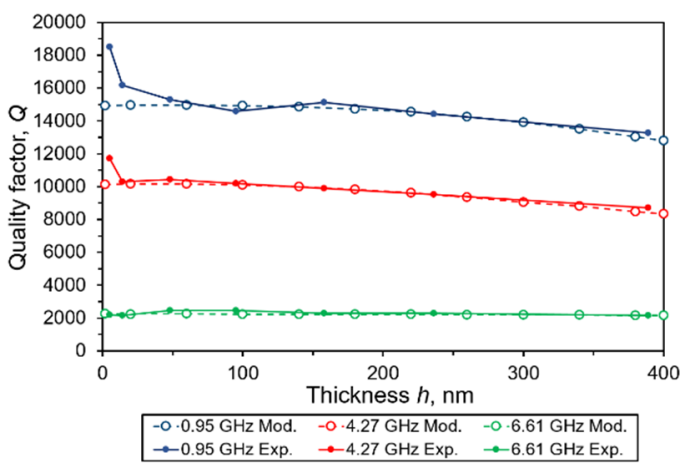

(b)

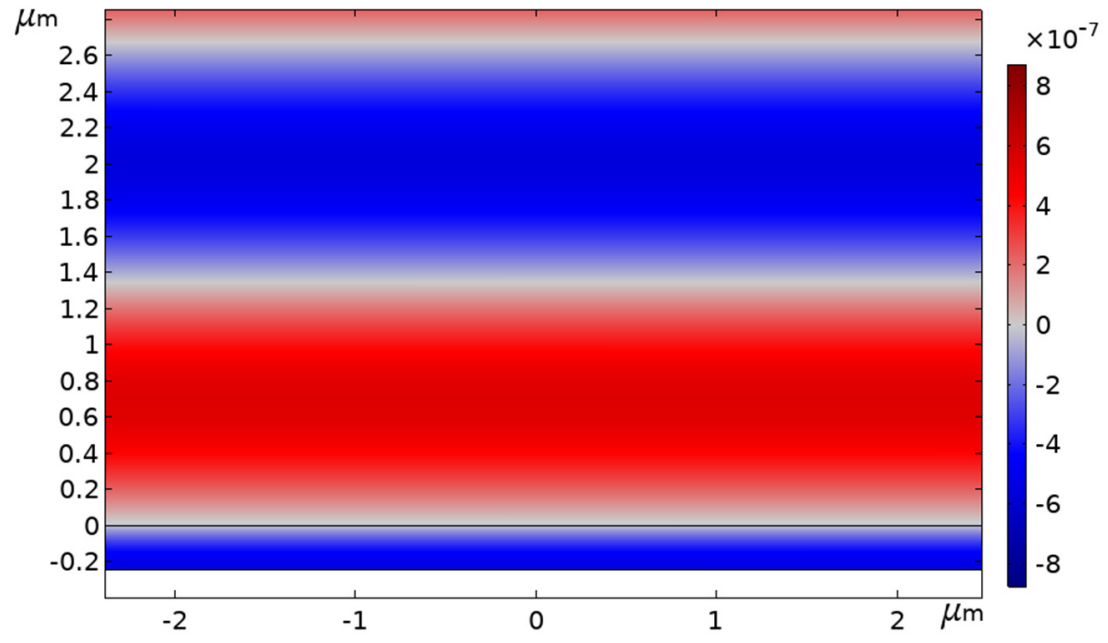

(c)

Figure 4. Relative frequency shift (a) and quality factor (b) vs. Mo film thickness measured for the microwave acoustic overtones taken at three operational frequencies of sensor \#B. Model distribution of the L-BAW vertical Y-component of elastic displacements when $\mathrm{h}=240 \mathrm{~nm}$ at $6.61 \mathrm{GHz}$ (c).

As a result of Mo film deposition, only a linearly proportional dependence of the relative frequency shift on film thickness up to $\sim 400 \mathrm{~nm}$ was observed for some operating frequencies up to $6.6 \mathrm{GHz}$, as shown in Figure 4a. Let us take into account the approximate equality $Z_{\mathrm{Mo}} \approx Z_{\text {diam }}$. In fact, in this case, one can talk about the elongation of a wave propagation path in the "diamond-Mo" composite structure only. Thus, the dependence of the relative frequency shift vs. thickness is proportional. The measured dependence turned out to be quite close to the forecast obtained using FEM analysis.

Figure $5 \mathrm{a}, \mathrm{b}$ represent the relative frequency shift and quality factor vs. the Pt film thickness measured for the microwave acoustic overtones taken at four operational frequencies of $4.16 \mathrm{GHz}, 6.52 \mathrm{GHz}, 12.20 \mathrm{GHz}$, and $19.19 \mathrm{GHz}$ of sensor \#C. At $4.16 \mathrm{GHz}$ and $6.52 \mathrm{GHz}$, monotonic and close to proportional dependences $\Delta f / f=f(h)$ can be observed. As in the case of Sc film deposition, there one can see the complicated dependencies $\Delta f / f=f(h)$ and $Q=f(h)$ at the $12.12 \mathrm{GHz}$ and $19.19 \mathrm{GHz}$ overtones. Figure $5 \mathrm{c}, \mathrm{d}$ demonstrate the model distribution of the L-BAW vertical Y-components of elastic displacements taken at the points of the inflections of curves $\Delta f / f=f(h)$ and extremums of the $Q=f(h)$ dependences. As an example, one can say that the $\lambda / 4$ and $\lambda / 2$ values fit within the Pt thicknesses as $\sim 45 \mathrm{~nm}$ and $\sim 90 \mathrm{~nm}$ at $19.19 \mathrm{GHz}$, respectively. Similar to the Sc film case, in the condition $h=\lambda / 2$, a bulk acoustic wave penetrates from diamond into the $\mathrm{Pt}$ film and then reflects from its free surface resonating in the film directly. The $Q$-factor is approximately the same as in the initial state of a sensor $(h=0)$. 


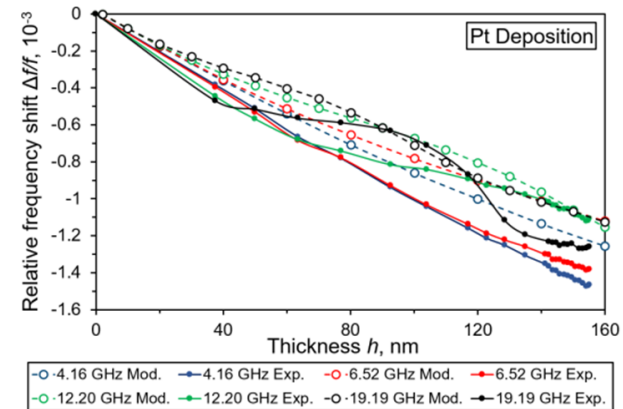

(a)

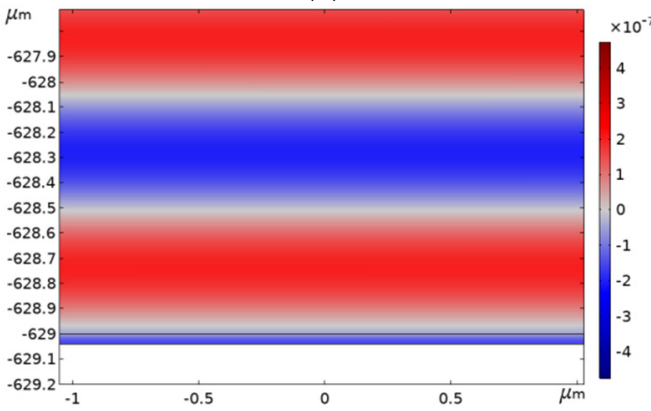

(c)

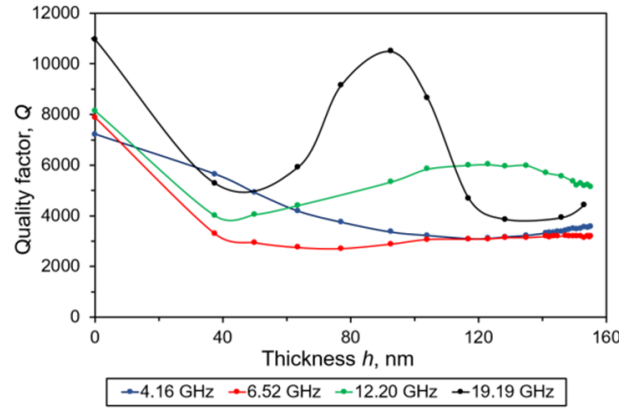

(b)

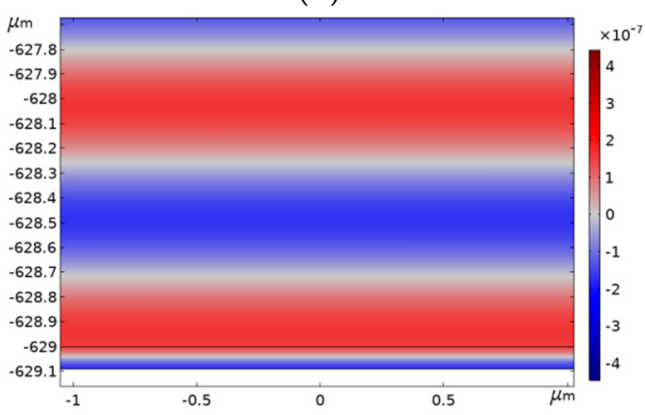

(d)

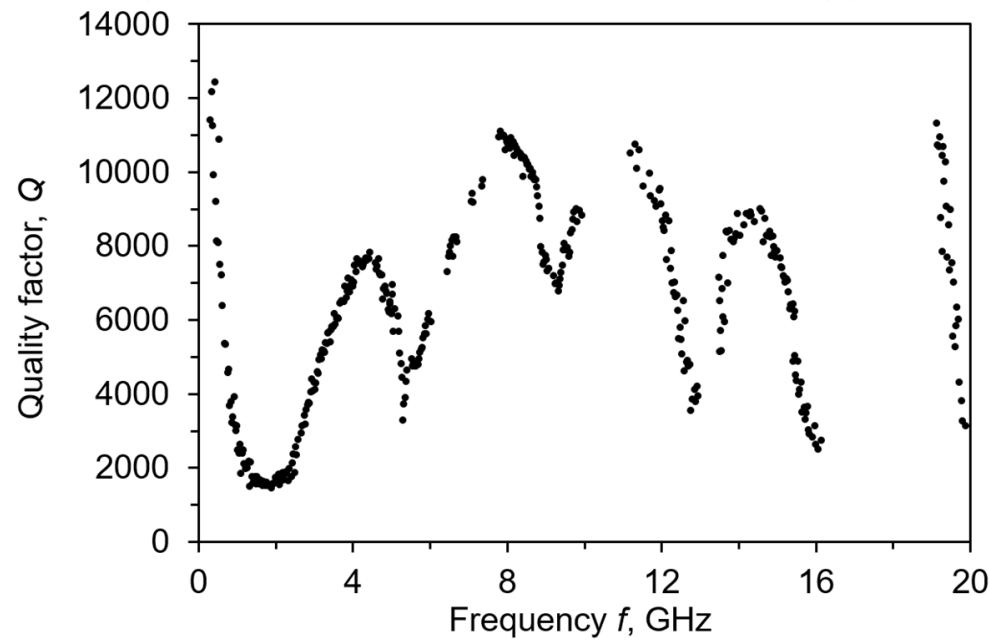

(e)

Figure 5. Relative frequency shift (a) and quality factor (b) vs. the Pt film thickness measured for the microwave acoustic overtones taken at four operational frequencies of sensor \#C. Model distribution of the L-BAW vertical Y-component of elastic displacements when $\mathrm{h}=45 \mathrm{~nm}$ (c) and $90 \mathrm{~nm}$ (d) at 19.19 GHz. (e) Preliminary measurement of the Q-factor frequency dependence.

\section{Discussion}

Theoretical and experimental results of microwave acoustic wave propagation in diamond-based multilayer piezoelectric structures as "Me1/(Al,Sc)N/Me2/(100) diamond/Me3" and "Me1/AlN/Me2/(100) diamond/Me3" under three metal film depositions were obtained. The relatively small decrease in the quality factor of diamond-based HBARs under the metal layer effect observed in a wide microwave band up to $20 \mathrm{GHz}$ could be qualified as an important result. Changes in operational resonant frequencies vs. film thickness were discovered to have sufficient distinctions.

From the comparison of the $\Delta f / f=f(h)$ dependences for the Sc, Mo, and Pt deposited films (Figures 3a, 4a and 5a), it follows that the HBAR-based sensor had the best sensitivity to changes in a Me3 film thickness nearby the points when $h_{\mathrm{Me} 3} \approx n \lambda / 4(n=1,3,5, \ldots)$ for metals with acoustic impedance satisfying $Z_{\mathrm{Me} 3}<Z_{\text {diam }}$. For metals with $Z_{\mathrm{Me} 3}>Z_{\text {diam }}$, 
the highest sensitivity was observed at the points when $h_{\mathrm{Me} 3} \approx m \lambda / 2(m=1,2,3, \ldots)$. The more the impedance of a film differs from the impedance of the diamond substrate, the higher the sensor's sensitivity is.

The explanation of $\Delta f / f=f(h)$ and $Q(h)$ dependences is based on the difference in the reflection coefficients $R$ of an elastic wave reflected on the substrate-film interface due to the difference in their acoustic impedances [25]. At $Z_{\mathrm{Me} 3}<Z_{\text {diam, }}$, when the acoustic wave reflects from a film in the condition $h_{\mathrm{Me} 3} \approx n \lambda / 4$, the reflection coefficient tends to zero, and the wave's phase changes by $180^{\circ}$. The waves that pass into the film experience multiple reflections from the substrate-film interface and the outer surface of a film. At the outer surface of the film, all these waves add up in phase. As a result, an increase in the amplitude of their oscillations occurs. This effect leads to significant energy dissipation under the repeated passage of a wave through the thickness of a film. In this case, the quarter-wave film becomes a strong absorber of sound waves. As a result, the decrease in the Q-factor of an HBAR-based sensor is observed experimentally (Figures $3 b$ and $5 b$ ).

Under the condition $Z_{\mathrm{Me} 3}>Z_{\text {diam }}$ at the points of $h_{\mathrm{Me} 3} \approx m \lambda / 2$, the $R$-coefficient for a wave reflected from a film into diamond substrate has the maximal value. In this case, this coefficient is equal to the $R$-coefficient for a wave reflected from the free substrate boundary. Since the transparency coefficient $T=1-R$ of the film drops to zero, there is a kind of "shutdown" of a film, according to Brekhovskikh [25]. As a result, the $Q$-factor of the HBAR-based sensor remains approximately the same as in the absence of a film (Figure $5 b$ ).

The slight decrease in $Q$-factor observed in the frequency range up to $20 \mathrm{GHz}$, excluding the abovementioned special points, is due to the fact that the $Q$-factor in HBAR is mainly determined by the ratio of stored energy to its dissipation over a period. Because the diamond substrate has the largest reserve of elastic energy, and the Landau-Rumer acoustic attenuation law is fulfilled in diamond beginning in the frequency range of $1.5-2 \mathrm{GHz}$, both these circumstances determine the relative stability of a $Q$-factor in such a wide frequency range. This is of great importance for the successful application of HBAR-based sensors on diamond substrates.

\section{Conclusions}

Enhanced experimental results including the $Q$-factor's dependences on the film thickness were introduced and discussed. The HBAR-based sensor's model was proposed and studied in detail using FEM. A complete explanation of the peculiarities of the BAW propagation in diamond-based MPSs under the influence of a certain metal film deposition was provided. The special points on the $\Delta f / f=f(h)$ and $Q(h)$ dependences for the Sc, Mo, and $\mathrm{Pt}$ films were highlighted. The search for enhanced sensitivity in an HBAR-based sensor was discussed in terms of both the above points and the relation between acoustic impedances of a substrate and film.

The diamond-based multilayer piezoelectric structures as sensory elements demonstrate clear potential owing to their operation in the microwave band up to $20 \mathrm{GHz}$, as well as the enhanced sensitivity, high chemical and biological inertness of the working diamond surface, resistance to temperature load, abrasive wear resistance, and possibility of multiple applications. The investigated diamond-based fifth layered piezoelectric structure should be considered as a prospective platform for developing a new generation of multipurpose sensors.

Author Contributions: Conceptualization, B.S. and V.P.; methodology, software, and visualization, G.K.; investigation, N.A. and A.S.; writing-original draft preparation and writing—review and editing, B.S.; funding acquisition, V.P. All authors read and agreed to the published version of the manuscript.

Funding: The work was done using the Shared Research Facilities "Research of Nanostructured, Carbon, and Superhard Materials" of the FSBI TISNCM. 
Conflicts of Interest: The authors declare no conflict of interest. The funders had no role in the design of the study; in the collection, analyses, or interpretation of data; in the writing of the manuscript, or in the decision to publish the results.

\section{References}

1. Schedin, F.; Geim, A.K.; Hill, E.W.; Blake, P.; Novoselov, K.S. Detection of individual gas molecules adsorbed on graphene. Nat. Mater. 2007, 6, 652-655. [CrossRef] [PubMed]

2. Johnson, L.; Gupta, A.K.; Ghafoor, A.; Akin, D.; Bashir, R. Characterization of vaccinia virus particles using microscale silicon cantilever resonators and atomic force microscopy. Sens. Actuators B Chem. 2006, 115, 189-197. [CrossRef]

3. Dantham, V.R.; Holler, S.; Kolchenko, V.; Wan, Z.; Arnold, S. Taking whispering gallery-mode single virus detection and sizing to the limit. Appl. Phys. Lett. 2012, 101, 043704. [CrossRef]

4. $\quad$ Ekinci, K.L.; Huang, X.M.H.; Roukes, M.L. Ultrasensitive nanoelectromechanical mass detection. Appl. Phys. Lett. 2004, 84, 4469-4471. [CrossRef]

5. Sauerbrey, G. Verwendung von Schwingquarzen zur Wägung dünner Schichten und zur Mikrowägung. Z. Physik. 1959, 155, 206-222. [CrossRef]

6. Lu, C.S.; Lewis, O. Investigation of film-thickness determination by oscillating quartz resonators with large mass load. J. Appl. Phys. 1972, 43, 4385-4390. [CrossRef]

7. Muratsugu, M.; Ohta, F.; Miya, Y.; Hosokawa, T.; Kurosawa, S.; Kamo, N.; Ikeda, H. Quartz crystal microbalance for the detection of microgram quantities of human serum albumin: Relationship between the frequency change and the mass of protein adsorbed Anal. Chem. 1993, 65, 2933-2937. [CrossRef] [PubMed]

8. Qiao, X.; Zhang, X.; Tian, Y.; Meng, Y. Progresses on the theory and application of quartz crystal microbalance. Appl. Phys. Rev. 2016, 3, 031106. [CrossRef]

9. Wen, W.; Shitang, H.; Shunzhou, L.; Minghua, L.; Yong, P. Enhanced sensitivity of SAW gas sensor coated molecularly imprinted polymer incorporating high frequency stability oscillator. Sens. Actuators B Chem. 2007, 125, 422-427. [CrossRef]

10. Lin, R.C.; Chen, Y.C.; Chang, W.T.; Cheng, C.C.; Kao, K.S. Highly sensitive mass sensor using film bulk acoustic resonator. Sens Actuators A Physical. 2008, 147, 425-429. [CrossRef]

11. Zhang, H.; Kim, E.S. Micromachined acoustic resonant mass sensor. J. Microelectromechanical Syst. 2005, 14, 699-706. [CrossRef]

12. Rey-Mermet, S.; Lanz, R.; Muralt, P. Bulk acoustic wave resonator operating at $8 \mathrm{GHz}$ for gravimetric sensing of organic films. Sens. Actuators B 2006, 114, 681-686. [CrossRef]

13. Wingqvist, G.; Bjurström, J.; Liljeholm, L.; Yantchev, V.; Katardjiev, I. Shear mode AlN thin film electro-acoustic resonant sensor operation in viscous media. Sens. Actuators B 2007, 123, 466-473. [CrossRef]

14. Mansfeld, G.D.; Alekseev, S.G.; Kotelyansky, I.M. Acoustic HBAR spectroscopy of metal (W, Ti, Mo, Al) thin films. In Proceedings of the 2001 IEEE Ultrasonics Symposium. Proceedings. An International Symposium (Cat. No.01CH37263), Atlanta, GA, USA, 7-10 October 2001; pp. 415-418. [CrossRef]

15. Rabus, D.; Friedt, J.M.; Ballandras, S.; Baron, T.; Lebrasseur, É.; Carry, É. High-overtone bulk-acoustic resonator gravimetric sensitivity: Towards wideband acoustic spectroscopy. J. Appl. Phys. 2015, 118, 114505. [CrossRef]

16. Sorokin, B.P.; Kvashnin, G.M.; Novoselov, A.S.; Bormashov, V.S.; Golovanov, A.V.; Burkov, S.I.; Blank, V.D. Excitation of hypersonic acoustic waves in diamond-based piezoelectric layered structure on the microwave frequencies up to 20 GHz. Ultrasonics 2017, 78, 162-165. [CrossRef] [PubMed]

17. Sorokin, B.P.; Telichko, A.V.; Kvashnin, G.M.; Bormashov, V.S.; Blank, V.D. Study of microwave acoustic attenuation in a multifrequency bulk acoustic wave resonator based on a synthetic diamond single crystal. Acoust. Phys. 2015, 61, 669-680. [CrossRef]

18. Sorokin, B.P.; Asafiev, N.O.; Kvashnin, G.M.; Scherbakov, D.A.; Terentiev, S.A.; Blank, V.D. Toward 40 GHz excitation of diamond-based HBAR. Appl. Phys. Lett. 2021, 118, 083501. [CrossRef]

19. Sorokin, B.P.; Kvashnin, G.M.; Asafiev, N.O.; Luparev, N.V. Microwave Acoustic Mass Sensor. RF Patent Application No. 2019128681, 18 June 2020.

20. Sorokin, B.P.; Kvashnin, G.M.; Novoselov, A.S.; Burkov, S.I.; Shipilov, A.B.; Luparev, N.V.; Aksenenkov, V.V.; Blank, V.D. Application of thin piezoelectric films in diamond-based acoustoelectronic devices. In Piezoelectricity-Organic and Inorganic Materials and Applications; Vassiliadis, S.G., Matsouka, D., Eds.; IntechOpen: Rijeka, Croatia, 2018; Chapter 2; pp. 15-41.

21. Sorokin, B.P.; Kvashnin, G.M.; Luparev, N.V.; Asafiev, N.O.; Scherbakov, D.A. Studying microwave acoustic sensors based on synthetic diamond substrates. Izvestiya Vysshikh Uchebnykh Zavedenii, Seriya Khimiya i Khimicheskaya Tekhnologiya 2020, 63, 63-70. (In Russian) [CrossRef]

22. Sorokin, B.; Kvashnin, G.; Asafiev, N.; Kravchuk, K.; Luparev, N.; Sotnikov, A. Microwave diamond-based HBAR as ultrathin film sensor. Pt deposition. In Proceedings of the 2020 Joint Conference of the IEEE International Frequency Control Symposium and International Symposium on Applications of Ferroelectrics (IFCS-ISAF), Keystone, CO, USA, 19-23 July 2020; pp. 1-4. [CrossRef]

23. Ebrahimi, F.; Dabbagh, A. Wave propagation analysis of embedded nanoplates based on a nonlocal strain gradient-based surface piezoelectricity theory. Eur. Phys. J. Plus 2017, 132, 449. [CrossRef] 
24. Ebrahimi, F.; Dabbagh, A. Wave Propagation Analysis of Smart Nanostructures; CRC Press: Boca Raton, FL, USA, 2019.

25. Brekhovskikh, L.M. Waves in Layered Media, 2nd ed.; Academic Press: New York, NY, USA; London, UK; Toronto, ON, Canada; Sydney, Australia; San Francisco, CA, USA, 1980; pp. 1-503. 